\title{
Clinical effectiveness of a skills training intervention for caregivers in improving patient and caregiver health following in-patient treatment for severe anorexia nervosa: pragmatic randomised controlled trial
}

\author{
Rebecca Hibbs, Nicholas Magill, Elizabeth Goddard, Charlotte Rhind, Simone Raenker, \\ Pamela Macdonald, Gill Todd, Jon Arcelus, John Morgan, Jennifer Beecham, Ulrike Schmidt, \\ Sabine Landau and Janet Treasure
}

\section{Background}

Families express a need for information to support people with severe anorexia nervosa.

\section{Aims \\ To examine the impact of the addition of a skills training intervention for caregivers (Experienced Caregivers Helping Others, ECHO) to standard care.}

\section{Method}

Patients over the age of 12 (mean age 26 years, duration 72 months illness) with a primary diagnosis of anorexia nervosa and their caregivers were recruited from 15 in-patient services in the UK. Families were randomised to ECHO (a book, DVDS and five coaching sessions per caregiver) or treatment as usual. Patient $(n=178)$ and caregiver $(n=268)$ outcomes were measured at discharge and 6 and 12 months after discharge.

\section{Results}

Patients with caregivers in the $\mathrm{ECHO}$ group had reduced eating disorder psychopathology (EDE-Q) and improved quality of life (WHO-Quol; both effects small) and reduced in-patient bed days (7-12 months post-discharge). Caregivers in the ECHO group had reduced burden (Eating Disorder Symptom Impact
Scale, EDSIS), expressed emotion (Family Questionnaire, FQ) and time spent caregiving at 6 months but these effects were diminished at 12 months.

\section{Conclusions}

Small but sustained improvements in symptoms and bed use are seen in the intervention group. Moreover, caregivers were less burdened and spent less time providing care. Caregivers had most benefit at 6 months suggesting that booster sessions, perhaps jointly with the patients, may be needed to maintain the effect. Sharing skills and information with caregivers may be an effective way to improve outcomes. This randomised controlled trial (RCT) was registered with Current Controlled Trials ISRCTN06149665

\section{Declaration of interest}

J.T. is a co-author of the book used in the $\mathrm{ECHO}$ intervention and receives royalties.

\section{Copyright and usage}

(c) The Royal College of Psychiatrists 2015. This is an open access article distributed under the terms of the Creative Commons Non-Commercial, No Derivatives (CC BY-NC-ND) licence.
Anorexia nervosa usually develops in early adolescence but over $50 \%$ of cases have an illness that persists for over 7 years and extends into adulthood. ${ }^{1}$ The treatment response differs according to the stage of illness. ${ }^{2}$ For example, family-based therapy is more effective in the early stage of illness. ${ }^{3}$ The absolute rate of full recovery is less than one-third overtime. ${ }^{4,5}$

Individuals with high levels of medical risk are managed by specialised in-patient care. The mortality, morbidity and service use and cost for in-patient care for anorexia nervosa are amongst the highest of all psychiatric disorders. ${ }^{6-8}$ Protracted support from both the state and/or family is needed for people with the severe enduring form of illness. ${ }^{9}$ Family members experience high levels of burden and distress ${ }^{10,11}$ and request information and help with their caregiving role. ${ }^{12-14}$ We have developed a skills training intervention (Experienced Caregivers Helping Others (ECHO)) to support the caregiving role at home based on caregivers' needs and our cognitive interpersonal model of anorexia nervosa. ${ }^{15,16}$

The aim of this study was to evaluate the impact of the addition of the ECHO intervention on patient and caregivers well-being following in-patient care. Families were randomised to receive ECHO plus treatment as usual (TAU) or TAU alone at the time patients were admitted to hospital. Caregiver and patient well-being was followed in the year following discharge. The trial investigated the following hypotheses regarding patient $(\mathrm{P})$ and caregiver $(\mathrm{C})$ outcomes. The primary patient hypothesis was that at 12-month followup, patients with caregivers allocated to ECHO would have a reduced rate of relapse compared to those allocated to TAU. The secondary hypothesis for patients was that patients with caregivers allocated to ECHO would sustain a higher body mass index (BMI) and quality of life and lower eating psychopathology (EDE-Q) and distress (Depression, Anxiety and Stress Scale-21 (DASS-21)) postrandomisation compared to those allocated to TAU. The primary hypothesis for caregivers was that caregivers allocated to ECHO would have less distress (depression, anxiety and stress DASS) in the year after discharge compared to those allocated to TAU. The secondary hypothesis for caregivers was that those who receive ECHO will report lower expressed emotion, accommodation and enabling, caregiving burden, time spent caregiving and improved quality of life post-randomisation compared to those allocated to TAU.

\section{Method}

This was a pragmatic, two-arm, multicentre parallel group randomised controlled trial (RCT). A detailed account of the trial 
protocol, including the description of the ECHO caregiver intervention, has been published. ${ }^{17}$ Consenting caregivers of patients who met eligibility criteria were randomly allocated to receive either ECHO (in addition to treatment as usual (TAU)) or TAU only. Guidance for the ECHO intervention was delivered by 'experienced' coaches (people with lived experience of eating disorders, $n=15$ ) and also (in order to have a sufficient numbers) post-graduate-level psychologists (without clinical training, $n=5$ ) who were specifically trained and supervised. Patients and their caregivers were recruited from 15 hospital units providing National Health Service (NHS) in-patient/day-patient care for people with eating disorders. Outcome measures were collected at baseline, discharge, and 6 and 12 months following discharge.

Ethics approval was granted by the Royal Free Hospital Ethics Committee (08/H0720/41) with site-specific ethics and governance approval for all participating sites. The study was adopted by the Mental Health Research Network (MHRN). Following informed consent (from caregiver and patient) and completion of baseline assessment (on admission to the treatment facility), caregivers of a patient were randomly allocated to ECHO or TAU using an online system. Randomisation was carried out independently by the King's Clinical Trials Unit at King's College London and employed minimisation with stratification factors, study site $(n=15)$ and disease severity (binary with the 'severe' category defined as one or both of body mass index (BMI) $<15$, presence of compensatory vomiting). The statisticians were masked to treatment allocation until adherence was measured.

\section{Setting, recruitment and participants}

Fourteen of the sites were eating disorder specialist in-patient wards in the UK (13 adults, 1 adolescent); one was a general (adolescent) psychiatric ward with eating disorder specialist staff. Four sites recruited day-patients as well as in-patients into the study. One of the specialist sites made local referrals to specialist eating disorder in-patient wards in the UK. Clinical Studies Officers (CSOs) from the MHRN supported recruitment of patients. Inclusion criteria: patients meeting the criteria of DSM-IV anorexia nervosa aged 12 years or above, able to speak and understand English. Exclusion criteria: no identified caregiver, patient/caregivers taking part in another treatment study or discharged from their in-patient stay before baseline assessment completed. In addition, participants with a severe comorbidity at time of admission (e.g. severe intellectual disability, physical illness, and psychosis) were not included in the study.

\section{Interventions}

Correspondence with caregivers on the randomisation outcome was by post. The ECHO group had a letter explaining that they would be contacted by their telephone coach and supporting documentation was sent (action/goal sheets, staging based on the transtheoretical model of change, ${ }^{18}$ frequently asked questions and troubleshooting for technical support). Those allocated to the TAU arm were given contact details for Beat, the leading UK eating disorder charity, and were offered access to the intervention on completion.

\section{ECHO}

Participants allocated to ECHO received this intervention as an addition to TAU. The materials were sent and the coaching begun immediately after randomisation. ECHO uses a skills training approach and consists of a book ${ }^{19}$ and five DVDs (three theoretical, two practical). The DVDs complement the information presented in the book with role plays and practical examples in using a motivational interviewing style of communication and strategies to reduce expressed emotion and accommodation. The information on the DVDs is presented visually with audio voiceover. A more detailed description of DVD content and the coaching input is described elsewhere. ${ }^{17,20}$ (A professionally produced version is available through the charity Succeed - www.succeedfoundation.org.)

In addition to the book and DVDs, the intervention package included five telephone coaching sessions per caregiver (up to 10 per family, e.g. mother and father). Single caregivers could receive up to 10 coaching calls. Participants were contacted by the coach within 2 weeks of receiving the material by post. Calls (of up to $40 \mathrm{~min}$ ) were made by appointment approximately two weekly. To be classified as completing the intervention a minimum of four calls (per family) were delivered or $75 \%$ of the book read. Information on coaches, their training and measurement of quality assurance is described in the published protocol. ${ }^{17}$ The majority of coaches (64\%) attained the recommended skill level for motivational interviewing. ${ }^{21}$

\section{Treatment as usual}

\section{In-patient or day-patient treatment}

The National Institute for Health and Care Excellence (NICE) guidelines have several Grade C recommendations about inpatient care. ${ }^{22}$ The Royal College of Psychiatrists has developed accreditation standards which detail how caregivers should be involved in in-patient treatment. ${ }^{23}$ For this study, day-patients were defined as patients who required non-residential intensive specialist treatment ( $\geq 4$ days a week).

\section{Aftercare}

The NICE guidelines specify that aftercare (focusing on eating behaviour) is provided for a year after discharge. This typically includes monitoring of physical risks, dietetic assessment and advice and some form of individual out-patient therapy. Cognitivebehavioural therapy, interpersonal psychotherapy or focal psychodynamic therapy are most commonly offered (see Results section for more detail).

\section{Assessment measures}

All participants (patients and caregivers) completed self-report assessments by post/telephone on admission to treatment unit, discharge from hospital and thereafter at 6 -and 12-month time point's post-discharge. For the year following discharge, patients additionally completed a short monthly assessment on core eating symptoms by telephone, email or post. Patient and caregiver primary and secondary outcomes are listed below. All measures have been validated in eating disorder populations and have good psychometric properties. See published protocol for more details. $^{17}$

\section{Patients' assessment measures}

Patients' baseline assessment includes the following:

- Sociodemographic features (a checklist used in pilot studies).

Patients' clinical outcome measures include the following:

- BMI (from clinical measures of height and weight).

- Eating Disorder Examination Questionnaire (EDE-Q). ${ }^{24}$ A self-report measure of eating disorder symptoms with good reliability and validity. 
- Depression, Anxiety and Stress Scale (DASS-21). ${ }^{25} \mathrm{~A}$ 21-item self-report measure validated in both clinical and non-clinical samples with good internal reliability.

- World Health Organization - Quality of Life Questionnaire (WHO-Quol). ${ }^{26} \mathrm{~A}$ self-report measure with good validity.

- The Client Service Receipt Inventory (CSRI); ${ }^{27}$ structured interview measuring use of specialist and generic health services.

Caregivers' assessment measures include the following:

- Burden: Eating Disorder Symptom Impact Scale (EDSIS): ${ }^{28}$ A 24-item self-report measure.

- DASS. As described above.

- Accommodation and Enabling Scale for Eating Disorders. ${ }^{29}$ A 33-item self-report measure.

- Family Questionnaire (FQ): this is a 20-item self-report measure assessing expressed emotion in caregivers using a 4-point Likert scale. ${ }^{30}$

- WHO-Quol. ${ }^{26}$

- The CSRI; ${ }^{27}$ As above but caregiver version measuring time spent caregiving.

\section{Statistical analysis}

All statistical analyses were based on the intention-to-treat principle; participants were analysed in the treatment arm to which they were randomised. The primary patient clinical outcome was time from discharge to relapse. A linear change was assumed between monthly measurements of BMI in order to interpolate the day on which two points were estimated to be lost. This variable was right censored since relapse might not have occurred by the end of the study period (1 year) or follow-up data for BMI could be missing. If five consecutive BMI measurements were missed, time to relapse was considered censored. The primary caregiver clinical outcome was distress at 12 months after discharge. To account for two primary outcomes, group differences on these outcomes were tested at a significance level of $2.5 \%$. Secondary caregiver and patient outcomes were continuous measures at discharge, 6-month or 1-year follow-up.

Time to relapse was defined as readmission to hospital due to their eating disorder or a drop of two points from discharge in BMI measured on a monthly basis (whichever came first) and analysed using Cox regression. Explanatory variables in this model were the variable of interest (treatment arm) and randomisation stratifiers (site and illness severity categories). The effect of treatment was estimated by the hazard ratio of relapse comparing ECHO with TAU.

The continuous secondary patient outcomes were analysed using linear mixed models. The dependent variable was the outcome at the respective time point (e.g. BMI at 12-month post-discharge) and (fixed) explanatory variables were given by treatment arm, baseline values of the variable under investigation (e.g. BMI at pre-randomisation) and randomisation stratifiers. In addition, the models can contain random intercepts for coaches in the ECHO group to allow for correlation in outcomes due to treatment being facilitated by the same coach. The models were used to estimate differences between treatment arms at each time point. Standardised treatment effect estimates were calculated by dividing estimated group differences by the common pre-randomisation standard deviation of the respective outcome.

Outcome variables contained considerable numbers of missing values; see Results section for details. We empirically identified a number of baseline variables that were predictive of missing values in outcome and also found that the primary caregiver not adhering to $\mathrm{ECHO}$ (coded ' 1 '=completed at least four coaching sessions or read at least $75 \%$ the coaching manual, ' 0 ' $=$ did not complete the intervention) was predictive of loss-to-follow up; see Results section for details. To allow for these processes driving missingness, in addition to allowing randomised group and values of the outcome under investigation at different time points being predictive of missingness, multiple imputation (MI), using chained equations ${ }^{31}$ with 100 imputations, was implemented. This allowed us to include predictors of missingness (including the post-randomisation variable adherence) in the imputation step without having to condition on these variables in the analyses models. $^{32}$

The analyses for continuous caregiver outcomes needed to encompass up to two caregivers per family (a nominated primary and second caregiver). Therefore, the analysis and imputation models described for patients were extended. First, the analysis models contained an additional random intercept that varied at the level of the patient to allow for similar outcomes for caregivers of the same patient. Second, to ensure that correlations were also reflected in the imputed values, imputations were carried out at the level of the patient allowing for two outcome variables - one for the primary caregiver and another for secondary caregiver (set to missing when there was no second caregiver, with resulting imputed values discarded before analysis)

Statistical analyses were carried out in Stata version 11. The user-contributed command ice was used for MI. ${ }^{31}$

\section{Results}

\section{Participant flow}

This study represents a collaboration of major UK eating disorder treatment centres and describes a large cross-section of severely ill patients with anorexia nervosa (37\% had regular objective binging and $30 \%$ had regular vomiting). In total, 178 patients and 268 caregivers were recruited. The CONSORT diagram for the study is shown in Fig. 1.

\section{Sample characteristics}

As expected from randomisation, patient and caregiver characteristics were well-balanced across groups (Table 1).

\section{Patients}

The descriptive details of the sample and the short-term effects of hospital treatment comparing symptom levels on admission and on discharge from in-patient care have been published. ${ }^{33}$

The majority of the patient group $(n=178)$ was significantly underweight (BMI $<15 \mathrm{~kg} / \mathrm{m}^{2}$ ) or had medical instability because of electrolyte problems. The mean age was 26 (s.d.=9) years. Eleven cases were from adolescent units; these were approximately equally distributed between the interventions (Table 1). The majority of cases $(n=123,69 \%)$ had an illness duration of more than 3 years and $47 \%(n=83)$ exceeding 6 years (enduring anorexia nervosa). Of the 11 adolescents: 8 had illness duration $<3$ years and 2 had illness duration $>3$ years. (This item was missing on one adolescent.) The median duration of the admission was 153.5 days (range 28-991). One patient remained an inpatient throughout the 2 years of the study. 
Assessed for eligibility ( $n=599)$

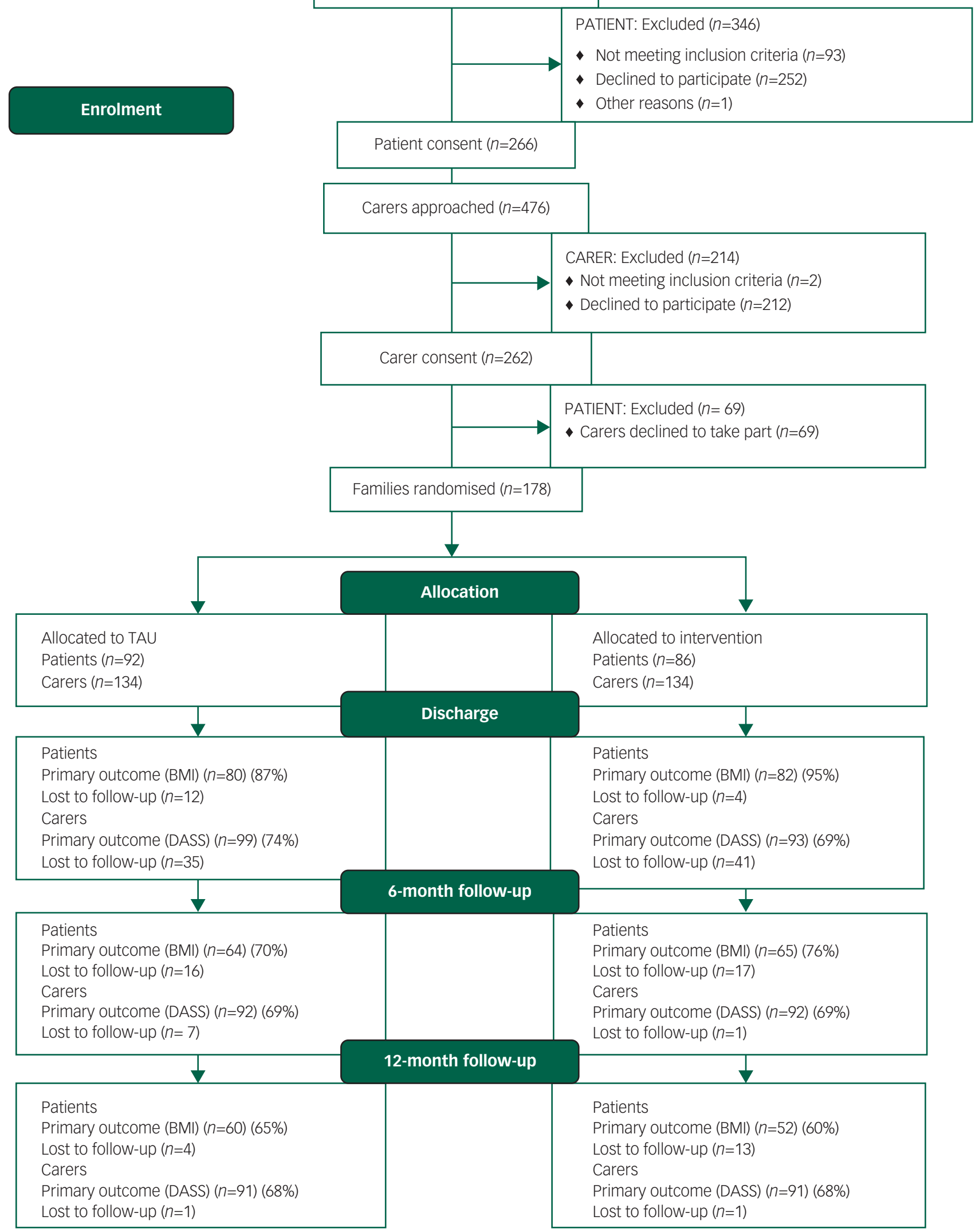

Fig. 1 Study consort diagram showing participant recruitment, allocation to TAU or TAU augmented with the ECHO intervention. TAU, treatment as usual; BMI, body mass index; DASS, Depression, Anxiety and Stress Scale. 
Table 1 Baseline characteristics by treatment group

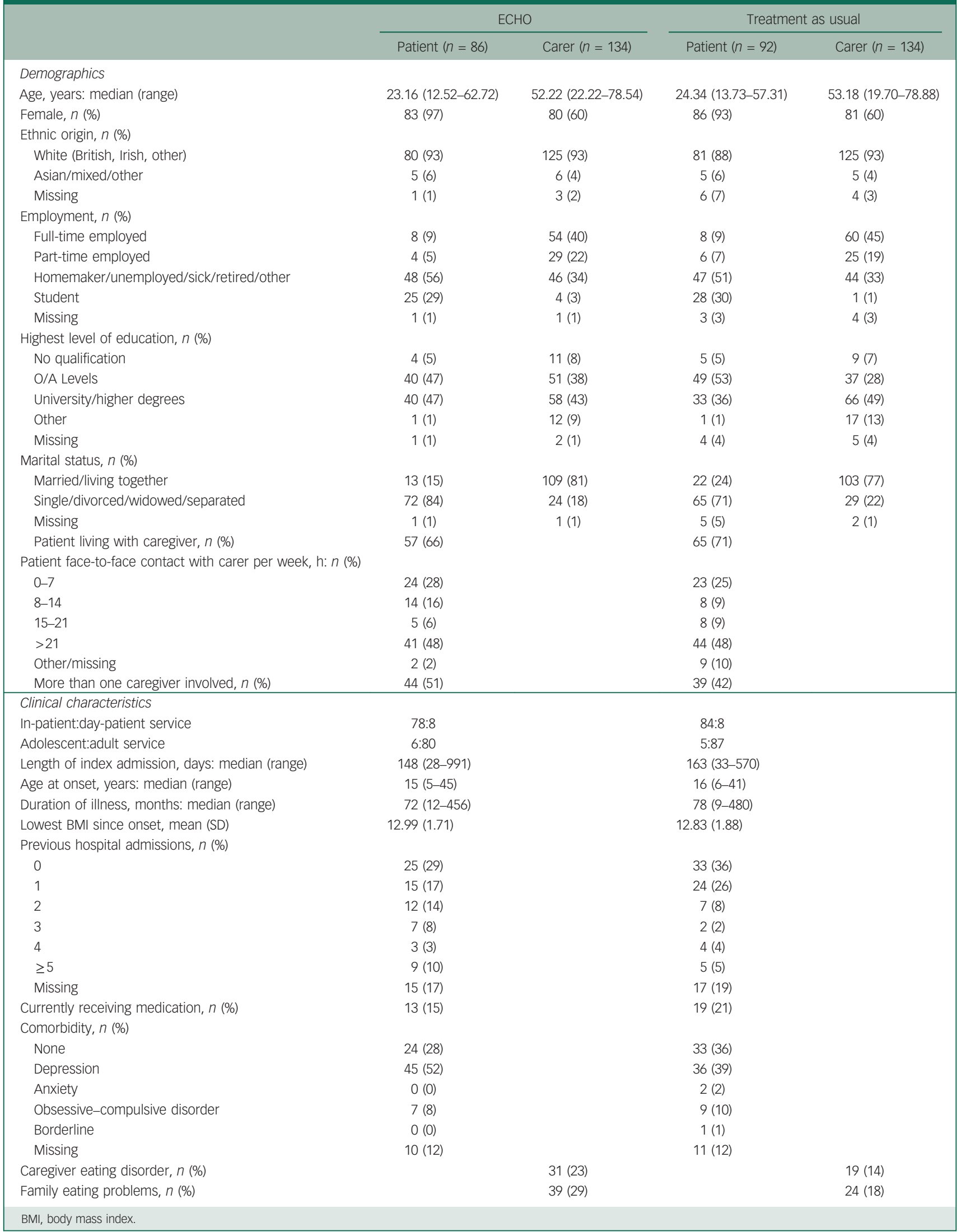

\section{Caregivers}

A total of 268 caregivers (178 primary caregivers, 90 secondary caregivers) were recruited (all were adults: 144 mothers, 81 fathers, 28 partners, 7 siblings, 5 friends, 3 other relatives). The mean number of caregivers per participant was 1.47 (range 1-3); see Table 1 for the number of caregivers involved in each group. The majority (69\%) lived with the patient with greater than $21 \mathrm{~h}$ of face-to-face contact time per week in $48 \%$ cases. 
Service characteristics. Only 11 patients were recruited from adolescent units and 16 from day-patient units. As expected, the numbers were equally distributed between trial arms. It is noteworthy that the adolescent units delivered family-based therapy before, during and after the in-patient admission $(88.9 \%$ of caregivers of patients admitted to hospital at an adolescent inpatient service attended family therapy during hospitalisation, compared with $24.1 \%$ of caregivers of patients admitted to hospital to an adult service).

Intervention adherence. Ninety-one caregivers (68\% of ECHO arm ( $15 \%$ missing information)) completed $75 \%$ of the coaching sessions and the manual.

\section{Important harms or unintended effects}

Two patients (one from each trial arm) died during the course of the study.

\section{Outcomes}

Fifty $(28 \%)$ patients at discharge, $57(32 \%)$ at 6 -month postdischarge and $66(37 \%)$ at 12 months had missing questionnaire outcome data. The number of caregivers with missing data was $86(32 \%)$ at discharge, $98(37 \%)$ at 6 months and $111(41 \%)$ at 12 months. Service use data (structured interview) were missing from $53(30 \%)$ of patients at 6 months and $55(31 \%)$ at 12 months.

Logistic regression was used to explore the relationships between a dependent variable that represented whether outcome data were present or missing at 12-month post-discharge and a number of baseline demographic and clinical variables, such as participant gender and lowest ever BMI, and also (post-treatment) adherence to ECHO. Any variable that showed a statistically significant association with the dependent variable was included in the imputation step of the MI procedure. Lowest ever BMI was predictive of missingness for patients' quality of life, eating disorder psychopathology (EDE-Q) and distress (DASS-21); age predicted missingness for patients' distress. It was also found that non-adherence with allocated therapy was strongly associated with missingness. The associations for caregivers $(P$-values as low as $P<0.001)$ were stronger than for patients (e.g. $P=0.04$ ).

Table 2 summarises the clinical outcomes and Table 3 provides estimated outcome differences between the two treatment arms for both patients and caregivers at all three time points.

\section{A comparison of patient clinical status in the year after hospitalisation between caregivers with and without ECHO intervention}

Eating disorder psychopathology (EDE-Q) and quality of life (WHO-Quol) were significantly better in the ECHO group at 6 months effect size (ES) $=-0.38$ and 0.38 respectively, but these differences were not significant at 12 -month post-discharge (Table 3). At 6 months after discharge we found eating disorder psychopathology (EDE-Q) among the ECHO group to be 0.47 points (95\% CI 0.03-0.92) less than in the TAU group. At the same time point, we estimated quality of life as measured on the WHO-Quol to be 0.91 points ( $95 \%$ CI $0.04-1.78$ ) higher in the ECHO group compared with the TAU group. Estimated differences in distress and BMI pointed towards a beneficial effect of $\mathrm{ECHO}$, but none of these effects could be shown to be statistically significant.

\section{A comparison of caregivers' well-being and burden in the year after hospitalisation between caregivers with and without $\mathrm{ECHO}$ intervention}

Although caregivers in the ECHO group reported fewer symptoms of distress than those in TAU, this was not statistically significant at any time point (Table 3). Caregiving burden (EDSIS), at discharge, was significantly lower in the ECHO group ( $\mathrm{ES}=-0.29$ ). Time spent caregiving $(E S=-0.34)$ and expressed emotion $(\mathrm{FQ}, \mathrm{ES}=-0.25)$ were significantly lower in the ECHO group at 6 months after discharge. At discharge, we estimated that caregivers' perceptions of eating disorder burden (EDSIS) in the ECHO group were 3.98 points ( $95 \%$ CI $0.16-7.80$ ) less compared with TAU. At 6 months after discharge we found that time spent caregiving in the ECHO group was 63\% (95\% CI 40-100) of that spent in the TAU group. At the same time point, we estimated expressed emotion (FQ) in the ECHO group to be 2.24 points (95\% CI 0.05-4.43) less on the FQ than in the TAU group. At 12 months, the difference between groups in terms of expressed emotion, accommodating and caregiving time was negligible and there was a small effect in favour of ECHO for mood, burden and quality of life.

\section{A comparison of patient service use between caregivers with and without ECHO intervention}

The ECHO group had a slightly shorter median duration of admission (median=148 days, range 28-991) compared with the TAU group (median=163 days, range 33-570) but this was not statistically significant (Mann-Whitney $U$-test, $Z=-0.88, P=0.38$ ). The readmission rate was $27 \%(n=23)$ in the ECHO group and $32 \%$ $(n=29)$ in the TAU group. Relapse in terms of readmission and/or fall in two BMI points occurred in $43 \%$ of the ECHO group and $52 \%$ of TAU. The median time to relapse for the ECHO group was 262 days and 240 days for TAU. Survival plots of the time to relapse showed two survival curves that were broadly similar.

A box and whisker plot in Fig. 2 depicts use of beds in the two conditions in the year post-discharge. In the TAU condition, there was a higher bed usage 7-12 months post-discharge than in the ECHO group (Mann-Whitney $U$-test, $\mathrm{Z}=-1.97, P=0.049$ ).

The summary of bed days, primary care and secondary care appointments in the 6-month and 12-month intervals following discharge are shown in Table 4. There was little difference between the groups in terms of usage of primary and secondary care. Only a small proportion of the sessions in both groups involved the family.

\section{Discussion}

The aim of this study was to evaluate whether giving caregivers a skills training intervention (ECHO) improved both patient and caregiver outcomes in the year following hospital admission. All patients fulfilled the World Health Organization's definition of severe malnutrition $^{34}$ (and the majority had an illness duration of more than 3 years). Patients whose caregivers received the ECHO intervention had reduced eating disorder psychopathology (EDEQ) and improved quality of life (WHO-Quol) at 6 months (both small effects) and reduced in-patient bed days (7-12 months postdischarge). Estimated differences in distress and BMI pointed towards a beneficial effect of ECHO, but none of these effects could be shown to be statistically significant. Caregivers in the ECHO group had a small/moderate reduction in caregiver burden and reduced expressed emotion and a greater reduction in their time caregiving 6 months after discharge but these changes were diminished at 12 months. We did not find statistically significant effects of ECHO in terms of our distal primary outcomes, patient relapse and caregiver distress, although differences were in the anticipated direction. The size of the change effects for all secondary outcomes for both caregiver and patient were small but all favoured ECHO. Fewer bed days were used in the ECHO group. 


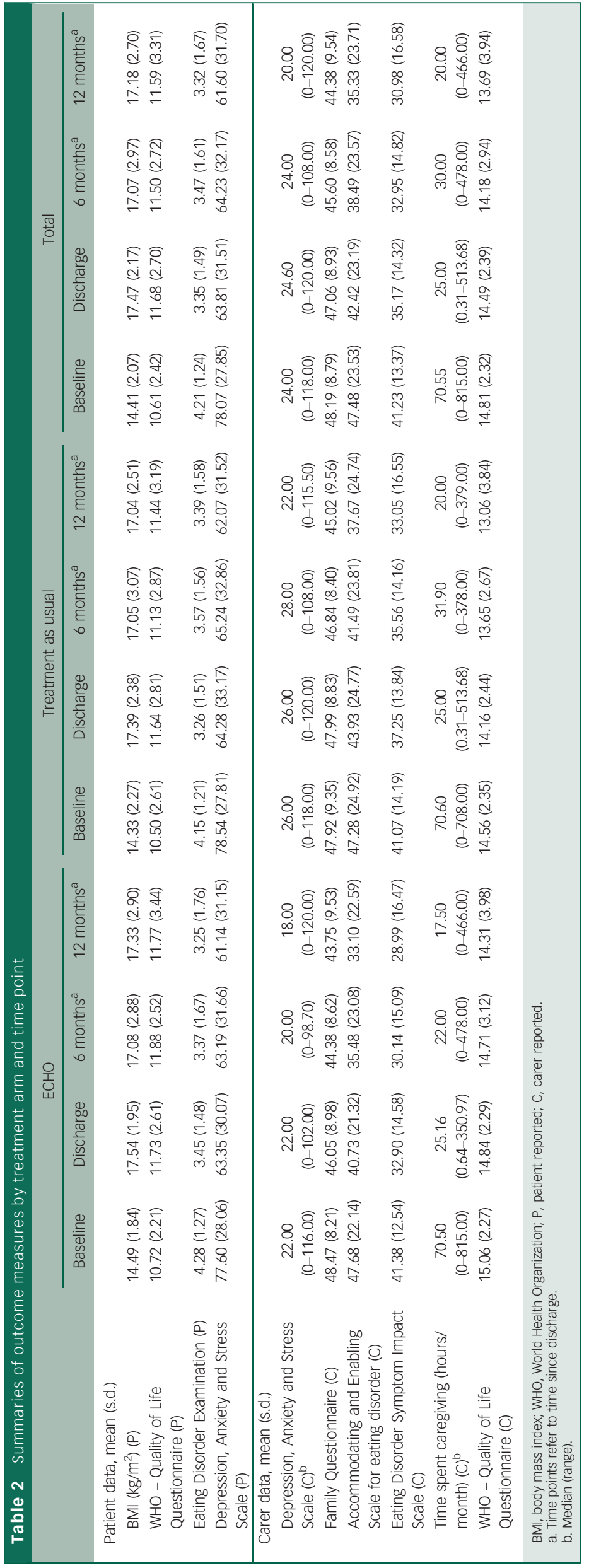




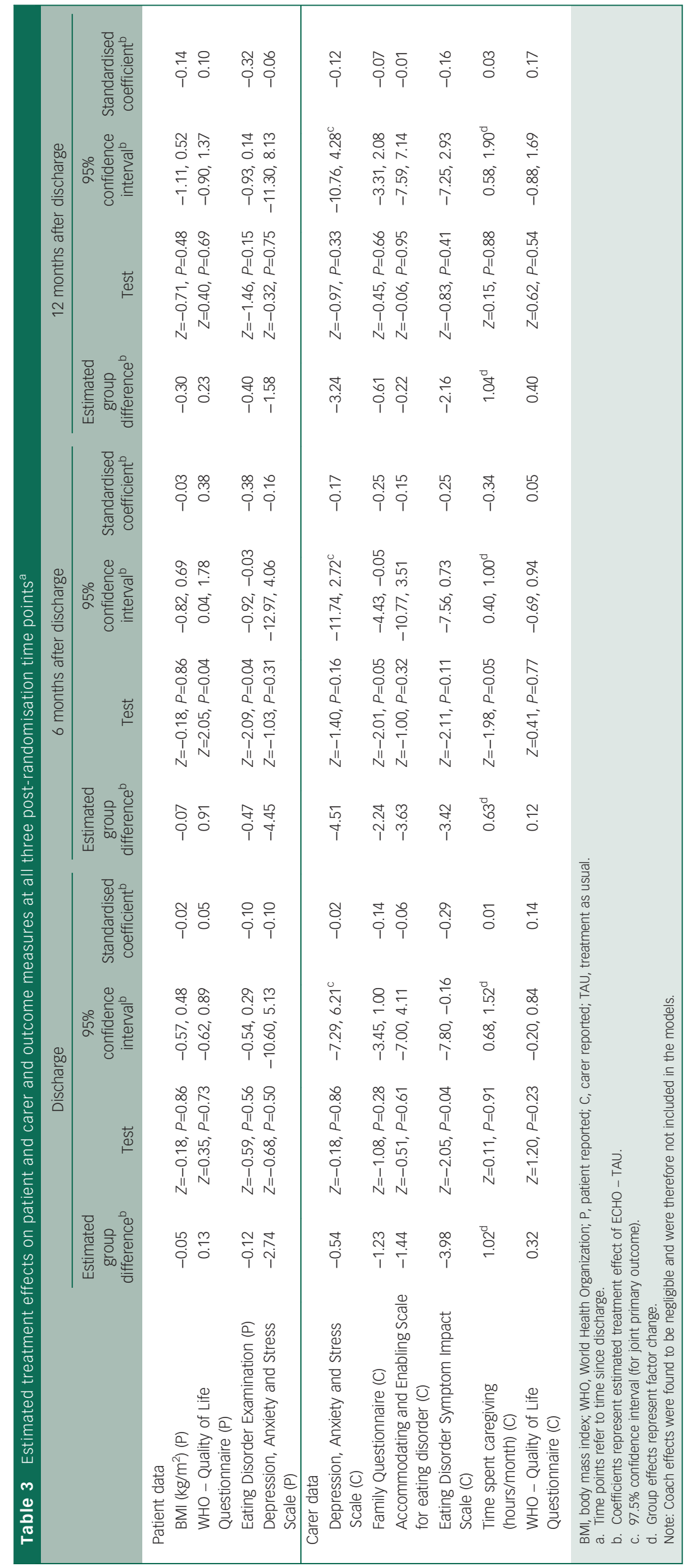




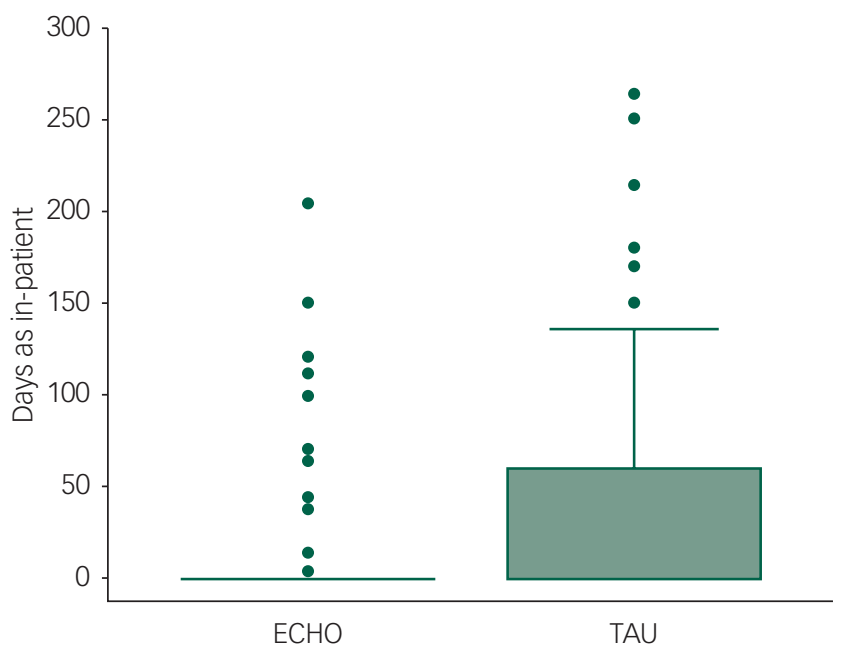

Fig. 2 Box and whisker plot (medians, 75 centile and outliers) for amount of time beds that were occupied in the year following discharge. TAU, treatment as usual.

Table 4 Eating disorder-related service use at 6-month and 12-month follow-up post-discharge by treatment group (ECHO experienced carer helping others $V$. TAU)

\begin{tabular}{|c|c|c|c|c|}
\hline \multicolumn{2}{|c|}{ Eating disorder-related service use } & $\mathrm{ECHO}$ & TAU & Total \\
\hline \multicolumn{5}{|l|}{ In-patient, days } \\
\hline 6 months & $N=124$ & 927 & 1276 & 2203 \\
\hline 12 months & $N=120$ & 499 & 1495 & 1994 \\
\hline \multicolumn{5}{|c|}{ GP/practise nurse, visits } \\
\hline 6 months & $N=124$ & 556 & 653 & 1209 \\
\hline 12 months & $N=123$ & 541 & 550 & 1092 \\
\hline \multicolumn{5}{|c|}{ Out-patient clinic, visits } \\
\hline 6 months & $N=123$ & 739 & 679 & 1418 \\
\hline 12 months & $N=119$ & 459 & 391 & 850 \\
\hline \multicolumn{5}{|c|}{ FBT/outreach/family support worker, visits } \\
\hline 6 months & $N=124$ & 33 & 115 & 148 \\
\hline 12 months & $N=120$ & 85 & 76 & 161 \\
\hline
\end{tabular}

The impact of the intervention on caregiver outcomes was limited to the first 6 months after discharge. This suggests that further booster sessions of family skills training throughout the year following admission or joint caregiver/patient sessions may be needed. It is noteworthy that the reduced bed use is not at the cost of greater burden and time caregiving from caregivers. Indeed the opposite effect occurred.

Interestingly, the size of the effect in terms of improvement of patient symptomatology remained present at 1 year suggesting that the indirect impact on patients may be more sustained despite less in-patient use. A 2-year follow-up of these patients that is in progress will be of interest to validate this conclusion.

The qualitative feedback (previously reported) also indicates that both patient and caregiver found the intervention helpful. ${ }^{35}$ Patients reported changes in their caregivers that include a greater understanding and awareness of the illness and improved coping abilities, better communication and reduced anxiety. Caregivers themselves noted improvements in their caregiving skills.

\section{Strengths}

This study used the strengths of a pragmatic randomised controlled design set within the majority of specialised eating disorder centres in England and so is relevant to current NHS England practice. This is one of the largest RCT studies within an in-patient setting and unique in considering the caregiver perspective. We added the intervention to TAU for pragmatic and economic reasons. We obtained information from three sources, patients, caregivers and services. The intervention was designed to be easy to disseminate and uses many principles advocated for a global application. ${ }^{36}$ As such, we used 'task sharing' with people with lived experience as we had found this expertise valued in our pilot studies. A small proportion of postgraduate psychologists were trained to increase the capacity to deliver the intervention. In a previously reported study, we found satisfactory levels of treatment fidelity in both groups with these different background experiences. ${ }^{21}$ Thus, given the low cost of the intervention, and the reduction in

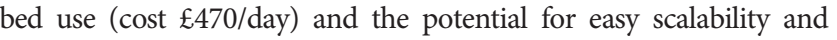
reach, we consider that this intervention is clinically relevant.

\section{Limitations}

Because of the research requirements (the agreement to involve caregivers), the sample is not necessarily representative of the intended target population. This might affect generalisability although in practice, because these materials are delivered directly to the caregiver, they could be part of standard information offered to caregivers in order to fulfil in-patient quality standards. ${ }^{23}$ In hindsight, a more eating disorder-related specific outcome such as caregiver burden or quality of life rather than caregiver distress may be more appropriate as the primary outcome for caregivers. Also in hindsight, the use of a binary/ time to event outcome 'relapse' such as our primary patient outcome was not useful for this patient group most of whom continue to meet diagnostic criteria at discharge. Other outcomes such as number of bed days, quality of life, BMI and/or the level of psychopathology would be clinically more meaningful.

Although there are standard quality criteria for in-patient care, the treatment ethos does vary between services. For example, some services involve caregivers more than others. We found that very little family support was offered as part of aftercare (on average 1 session per case). It is noteworthy that the adolescent units delivered family-based therapy before, during and after the in-patient admission. ${ }^{21}$ It is possible that this may have diluted the effect for these 11 families or it may even been harmful if the messages delivered were contradictory. Work is in progress to examine this possibility. This variability within the TAU condition may have decreased the size of group effect but there would be no bias in its estimation as the trial design was stratified by site. Further work to examine the factors that may influence the overall outcome will be of interest.

\section{Clinical implications}

A low-intensity skills-sharing intervention for the caregivers of patients with anorexia nervosa admitted for hospital care produced a small decrease in caregiver burden, expressed emotion and time spent caregiving at 6-month post-discharge but with less of an effect later. There was a small but sustained improvement in in-patients' quality of life and clinical symptoms. In-patient bed use 6-12 months after admission was reduced. This study illustrates how involving the family during in-patient care for adults as well as children can have benefits for both patient and caregiver. However, this form of augmentation of skills sharing for caregivers probably needs to be continued during the aftercare maintenance phase, particularly as caregivers may be the primary source of support for the individual during this time. The intervention has the potential to be easily disseminated and there may be a synergy in combining this with a skills-based intervention for patients themselves. Further work investigating the optimisation 
of in-patient care and after care for people with severe enduring anorexia nervosa is needed. It is noteworthy that in current NHS practice, in-patient care is a palliative intervention, only partially restoring weight. Although on average, the treatment gains are maintained in the year post-discharge, most patients remain severely ill. This poor outcome and the two deaths within the study highlight the need for better treatment for this patient group.

Rebecca Hibbs, MSc, PhD, Department of Psychological Medicine, Section of Eating Disorders, King's College London, Institute of Psychiatry, Psychology and Neuroscience (IOPPN), London, UK; Nicholas Magill, MSc, Biostatistics Department, King's College London, IOPPN, London, UK; Elizabeth Goddard, PhD, Department of Psychological Medicine, Section of Eating Disorders, King's College London, IOPPN, London,

UK; Charlotte Rhind, MSc, Department of Psychological Medicine, Section of Eating Disorders, King's College London, IOPPN, London, UK; Simone Raenker, PhD, Department of Psychological Medicine, Section of Eating Disorders, King's College London, IOPPN, London, UK; Pamela Macdonald, PhD, Department of Psychological Medicine, Section of Eating Disorders, King's College London, IOPPN, London,

UK: Gill Todd, RMN, MSC, South London and Maudsley NHS Foundation Trust, London,

UK; Jon Arcelus, MD, PhD, Eating Disorders Service, Leicestershire Partnership NHS Trust, Leicester, UK; John Morgan, MD, PhD, Yorkshire Centre for Eating Disorders, Leeds, UK; Jennifer Beecham, PhD, Personal Social Services Research Unit (PSSRU), London School of Economics and Political Science, London, UK: Ulrike Schmidt, MD, PhD, Department of Psychological Medicine, Section of Eating Disorders, King's College London, IOPPN, London, UK; Sabine Landau, PhD, Biostatistics Department, King's College London, IOPPN, London, UK; Janet Treasure, MD, PhD, Department of Psychological Medicine, Section of Eating Disorders, King's College London, IOPPN, London, UK

Correspondence: Janet Treasure, Institute of Psychiatry, Psychology and Neuroscience (IOPPN), King's College London, 103 Denmark Hill, London SE5 8AF, UK. E-mail: janet.treasure@kcl.ac.uk

First received 28 Jan 2015, final revision 27 May 2015, accepted 14 Jul 2015

\section{Acknowledgements}

We thank all families who participated and to the staff on the in-patient units that facilitated the study. We would also like to thank the Mental Health Research Network whose CSOs facilitated recruitment.

This article presents independent research commissioned by the National Institute for Health Research (NIHR) under its Programme Grants for Applied Research scheme (reference number RP-PG-0606-1043). Janet Treasure, Ulrike Schmidt and Sabine Landau received salary support from the NIHR Biomedical Research Centre at South London and Maudsley NHS Foundation Trust and King's College London. The views expressed are those of the author(s) and not necessarily those of the NHS, the NIHR or the Department of Health.

Our great thanks go to our experienced coaches who gave of their time and expertise to be trained in and to deliver the coaching: SnitaAhir-Knight, Leigh Best, Yvonne Boughton, Russell Cooper, Jocelyn de Guzman, Suzi Doyle, Amy Harrison, Jenny Langley. Melinda Waldrum, Esther Ritchie, Yvonne Round, Marjenah Shahabi, Jilly Shipway and Clare Walker This paper is in memory of Charlotte Bevan, a mother caregiver who strove to improve the support and skills of caregivers.

We also thank the principal investigators who are involved in the recruitment of participants at in-patient/day-patient sites: L. Whitehead, Cotswold House, Oxford; D. Robertson, Barberry Unit, Birmingham; A. Ayton, Darwin Centre, North Staffordshire S. Sharma, Cheadle Royal Hospital, Manchester; K. Moore, Kinver Centre, South Staffordshire: B. Bamford, St George's, London; N. Boughton,Cotswold House, Oxford; F. Connan, St Vincent's Clinic, London; K. Goss, Coventry; B. Laszlo, Haldon Unit, Exeter; C. SchreiberKounine,STEPS, Bristol; H. Lacey, St George's, London.

\section{Appendix: NHS England sites}

1. Barberry Unit, Birmingham

2. Bethlem Royal Hospital, London

3. Brandon Unit, Leicester

4. Cheadle Royal Hospital, Manchester

5. Cotswold House, Marlborough

6. Cotswold House, Oxford

7. Coventry

8. Darwin Centre, North Staffordshire

9. Haldon Unit, Exeter

10. Highfield Unit

11. Kinver Centre, South Staffordshire

12. Seacroft Hospital, LPFT

13. STEPS, Bristol

14. St George's, London

15. Vincent's Square, London

\section{References}

1 Stoving RK, Andries A, Brixen K, Bilenberg N, Horder K. Gender differences in outcome of eating disorders: a retrospective cohort study. Psychiatry Res 2011; 186: 362-6.

2 Treasure J, Stein D, Maguire S. Has the time come for a staging model to map the course of eating disorders from high risk to severe enduring illness? An examination of the evidence. Early Interv Psychiatry 2015; 9: 173-84.

3 Fisher CA, Hetrick SE, Rushford N. Family therapy for anorexia nervosa. Cochrane Database Syst Rev 2010; 14: 4

4 Eddy KT, Gray EK. Is seeking a panacea for anorexia nervosa a fool's errand? Long-term outcomes of family-based treatment. I Am Acad Child Adolesc Psychiatry 2014; 53: 1150-2.

5 Le Grange D, Lock J, Accurso EC, Agras S, Darcy A, Forsberg S, et al. Relapse from remission at two-to-four follow-up in two treatments for adolescent anorexia nervosa. J Am Acad Child Adolesc Psychiatry 2014; 53: 1162-7.

6 Chesney E, Goodwin GM, Fazel S. Risks of all-cause and suicide mortality in mental disorders: a meta-review. World Psychiatry 2014; 13: 153-60.

7 Hoang U, Goldacre M, James A. Mortality following hospital discharge with a diagnosis of eating disorder: national record linkage study, England, 2001-2009. Int J Eat Disord 2014; 47: 507-15.

8 Green BH, Griffiths EC. Hospital admission and community treatment of mental disorders in England from 1998 to 2012. Gen Hosp Psychiatry 2014; 36: 442-8.

9 Hjern A, Lindberg L, Lindbald F. Outcome and prognostic factors for adolescent female in-patients with anorexia nervosa: 9 to 14 year follow-up. Br J Psychiatry 2006; 189: 428-32.

10 Anastasiadou D, Medina-Pradas C, Sepulveda A, Treasure J. A systematic review of family caregiving in eating disorders. Eat Behav 15: 464-77.

11 Zabala M, Macdonald P, Treasure J. Appraisal of caregiving burden, expressed emotion and psychological distress in families of people with eating disorders: a systematic review. Eur Eat Disord Rev 2009; 17: 338-49.

12 De Zwaan M, Zipfel S, Herzog W, Herpertz-Dahlmann B, Konrad K, Hebebrand J, et al. EDNET - eating disorders diagnostic and treatment network. Psychother Psychosom Med Psychol 2009; 59: 110-16.

13 Graap H, Bleich S, Herbst F, Trostmann Y, Wancata J, De Zwaan M. The needs of carers of patients with anorexia and bulimia nervosa. Eur Eat Disord Rev 2008; 16: 21-9.

14 Haigh R, Treasure J. Investigating the needs of carers in the area of eating disorders: development of the Carers' Needs Assessment Measure (CaNAM). Eur Eat Disord Rev 2003; 11: 125-41.

15 Schmidt U, Treasure J. Anorexia nervosa: valued and visible. A cognitiveinterpersonal maintenance model and its implications for research and practice. Br J Clin Psychol 2006; 45: 343-66.

16 Treasure J, Schmidt U. The cognitive-interpersonal maintenance model of anorexia nervosa revisited: a summary of the evidence for cognitive, socioemotional and interpersonal predisposing and perpetuating factors. J Eat Disord 2013; 1: 13.

17 Goddard E, Raenker S, Macdonald P, Todd G, Beecham J, Naumann U, et al. Carers' assessment, skills and information sharing: theoretical framework and trial protocol for a randomised controlled trial evaluating the efficacy of a complex intervention for carers of inpatients with anorexia nervosa. Eur Eat Disord Rev 2013; 21: 60-71.

18 Prochaska J, DiClemente C. The Transtheoretical Approach: Crossing the Traditional Boundaries of Therapy. Dow Jones Irwin, 1984.

19 Treasure J, Smith G, Crane A. Skills-based Learning for Caregivers of a Loved One with an Eating Disorder: The New Maudsley Method. Routledge, 2007.

20 Sepulveda A, Lopez C, Macdonald P, Treasure J. Feasibility and acceptability of DVD and telephone coaching-based skills training for carers of people with an eating disorder. Int J Eat Disord 2008; 41: 318-25.

21 Macdonald P, Hibbs R, Rhind C, Harrison A, Goddard E, Raenker S, et al. Disseminating skills to carers of people with eating disorders: an examination of treatment fidelity in lay and professional carer coaches. Health Psychol Behav Med 2014; 2: 555-64.

22 National Institute for Health and Care Excellence. Guidelines for Eating Disorders: Core Interventions in the Treatment and Management of Anorexia Nervosa, Bulimia Nervosa, and Related Eating Disorders. NICE, 2004.

23 Cresswell J, Beavon M, Robinson H. Standards for Adult Inpatient Eating Disorder Service. Royal College of Psychiatrists, 2013.

24 Luce $\mathrm{KH}$, Crowther $\mathrm{JH}$. The reliability of the Eating Disorder Examination self-report questionnaire version (EDE-Q). Int J Eat Disord 1999; 25: 349-51.

25 Lovibond SH, Lovibond PF (eds). Manual for the Depression Anxiety Stress Scales (DASS): Psychology Foundation Monograph. University of New South Wales, 1993. 
26 WHOQOL. Development of the World Health Organization WHOQOL-BREF quality of life assessment. Psychol Med 1998; 28: 551-8.

27 Beecham J, Knapp M. Costing psychiatric interventions. In Measuring Mental Health Needs(2nd edn) (ed. G. Thornicroft): 200-24. Gaskell, 2001.

28 Sepulveda A, Whitney J, Hankins M, Treasure J. Development and validation of an Eating Disorder Symptom Impact Scale (EDSIS) for carers of people with eating disorders. Health Qual Life Outcomes 2008; 6: 1-9.

29 Sepulveda AR, Kyriacou O, Treasure J. Development and validation of the accommodation and enabling scale for eating disorders (AESED) for caregivers in eating disorders. BMC Health Serv Res 2009; 9: 171

30 Wiedemann G, Rayki O, Feinstein E, Hahlweg K. The family questionnaire: development and validation of a new self-report scale for assessing expressed emotion. Psychiatry Res 2002; 109: 265-79.

31 Royston P. Multiple imputation of missing values. Stata J 2004; 4: 227-41.
32 Sterne JA, White IR, Carlin JB, Spratt M, Royston P, Kenward MG, et al. Multiple imputation for missing data in epidemiological and clinical research: potential and pitfalls. BMJ 2009; 338: b2393.

33 Goddard E, Hibbs R, Raenker S, Salerno L, Arcelus J, Boughton N, et al. A multicentre cohort study of short term outcomes of hospital treatment for anorexia nervosa in the UK. BMC Psychiatry 2013; 13: 287.

34 World Health Organization. Physical Status: The Use and Interpretation of Anthropometry. WHO, 1995

35 Macdonald P, Rhind C, Hibbs R, Goddard E, Raenker S, Todd G, et al. Caregivers' Assessment, Skills and Information Sharing (CASIS) trial: a qualitative study of the experiential perspective of caregivers and patients. Eur Eat Disord Rev 2014; 22: 430-8.

36 Fairburn CG, Patel V. The global dissemination of psychological treatments: a road map for research and practice. Am J Psychiatry 2014; 171: 495-8. 